\title{
An Architecture For Web-Based Post-Sales Service In A Flexible Manufacturing Environment
}

\author{
Weidong Zhang ${ }^{1}$, Frans Coenen ${ }^{2}$ and Paul Leng ${ }^{2}$ \\ ${ }^{1}$ School of Computer Science, University of Windsor, Canada N9B 3P4 \\ ${ }^{2}$ Department of Computer Science, University of Liverpool, UK L69 7ZF
}

\begin{abstract}
Providing effective and efficient post-sales services is a significant issue facing industries operating in a flexible environment. The main problem is that the great diversity of the potential product range may make it impracticable to provide conventional support documentation for all versions offered. In this paper we explore some approaches of web-based information systems to support post-sales services in a customised manufacturing context. The major objective is to integrate various new technologies of Internet, Database and Expert Systems, such as JavaServer Page and Servlet Technology, JDBC, and Model-Based Methods, to supply on-line maintenance and diagnostic support to field service engineers.
\end{abstract}

\section{INTRODUCTION}

One aspect of commerce for which the Internet may be especially relevant is in the provision of post-sales services. It is becoming increasingly common for manufacturing industries to tailor their operation to be more in line with their customer requirements than (say) 10 years ago. That is to say that instead of offering a range of (say) 6 versions of a product, starting with a "deluxe" model at the top and working down to an "economy" model at the bottom; individual customisation is offered for the entire product range. This serves to increase the desirability of the product and (it is hoped) will encourage a corresponding increase in sales. There are, however, a number of difficulties associated with this approach, especially in respect of post-sales service.

For example, when perhaps 10,000 versions of a product are on offer, the storage of data concerning each version becomes problematic. More seriously the quantity of maintenance data required for an appropriate level of after sales service becomes 
increasingly difficult to 1) provide (in an effective manner) and 2) update. This is exacerbated when, as is often the case, the manufacturer is operating in a global market place. Thus although after sales services (i.e. maintenance) are seen as an important issue with respect to customer loyalty, there are issues concerning the provision of appropriate maintenance data that need to be addressed.

The issue of the provision and updating of maintenance information for manufacturers of the form described above is the principal concern of this paper. The aim of the paper is thus to provide a mechanism whereby maintenance information can be provided in a globally effective manner in such a way that it is both current and correct. In the delivery of field service information, the service agent in the field will hope to obtain information on demand, with minimal delay, after quoting only a brief product reference, ideally a unique identification found on the product. This information will be delivered by the product manufacturer and/or the parts suppliers. The obvious medium for the provision of the desired data is the Internet. However there are a number of issues to be considered. For instance the nature of the desired maintenance information, the presentation of the data, and the generation of the required information.

The rest of the paper is arranged as follows: 2. System Scenario, which identifies the basic entities and information involved in the proposed system; 3. Related Work, which reviews the previous research and development in related fields; 4. System Architecture, which specifies the system processes, system components and their relationships; 5. System Implementation, which describes the implementation architecture and the underlying techniques; and 6. Discussion and Conclusion, which discusses related issues and summaries the main findings and future work.

\section{SYSTEM SCENARIO}

In the delivery of field service information, three key entities are involved (see Figure 1). First, the service agent in the field will hope to obtain information on demand, with minimal delay, after quoting only a brief product reference, ideally a unique identification found on the product. This information will be delivered by the product manufacturer and/or the parts suppliers. In a conventional environment, it would be possible for the relevant data to be extracted from the product catalogue directly. In the flexible context we are considering, however, it will be necessary to create the information required by reference to the product design data and, thence, to data relating to the components of the product. The kinds of information delivered could be very diverse; we will briefly consider some of the more important categories. 


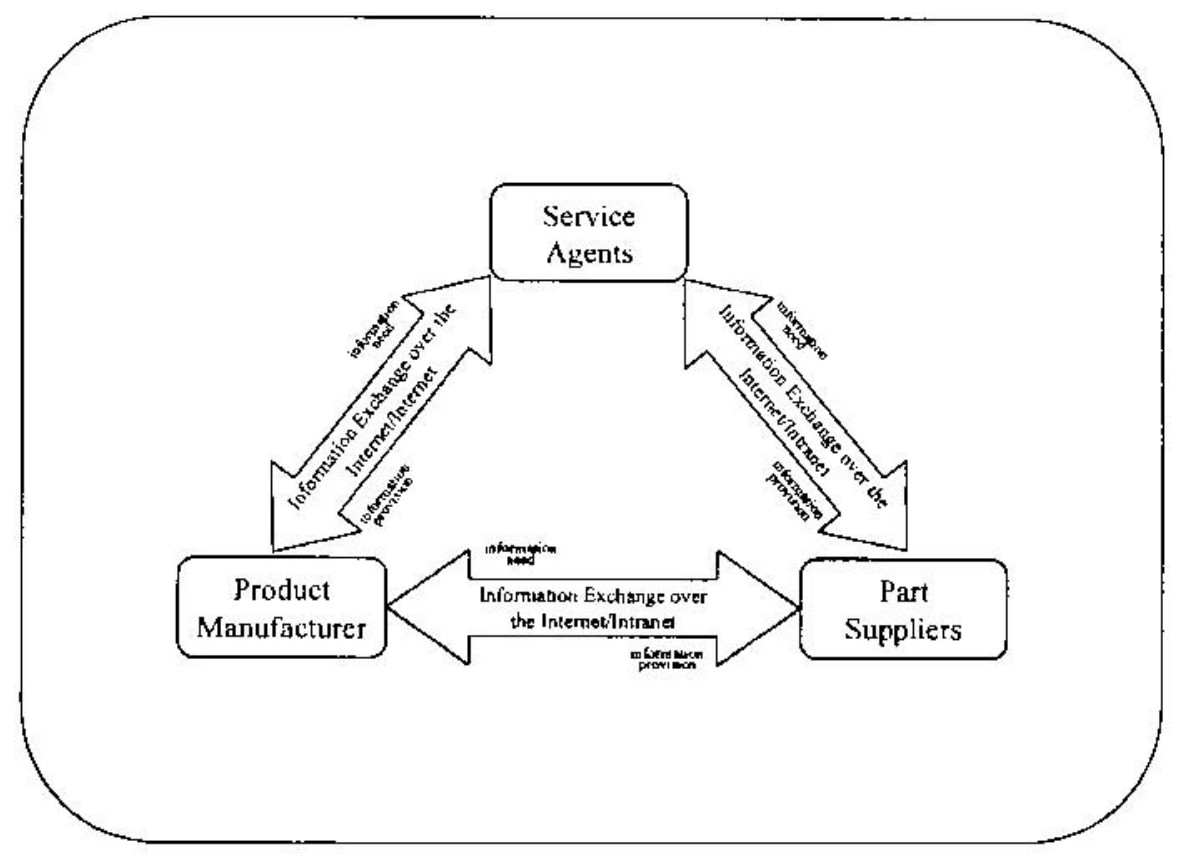

Figure 1. System scenario

\subsection{Textual and other descriptive data}

Usually, simple text descriptions will be a central feature of the information provided. In an online context, the presentation of text can be augmented and structured using hypermedia techniques [5], and the information incorporated may then include other non-textual data, including photographs, video clips, and animations. In an engineering context, detailed product information in the form of plans, engineering drawings, and design data will also be essential, possibly including access to a product database.

\subsection{Product modelling}

Even when dynamically generated and augmented by non-textual information, product documentation is essentially an organisation of data into a convenient and manageable form. Product modelling goes beyond this, in presenting information on the appearance and behaviour of the product in a form which allows the user (in this case, the service agent) to interact with the description: for example, to configure the model with locally-applicable data, to conduct experiments, and to focus on specific features. Virtual Reality (VR) languages, for example VRML [4] and Java3D [14], 
allow product models to be built which incorporate 3D graphics and enable "fly through" simulation to illustrate behaviour.

\subsection{Expert knowledge}

Much of the information used by service engineers, as with other skilled professionals, may take the form not just of factual data but also of accumulated expertise. As in other domains such as medicine in which diagnosis is central, Knowledge-Based Systems may be expected to have a role here. Production rule systems [3] have been favoured by the Expert System community for many years, particularly for diagnostic systems which may often be represented readily in terms of "if-then-else" rules, and there continues to be active research into rule-based diagnosis in many contexts [12].

\subsection{Case-based data}

Another important basis for fault diagnosis in the field is the use of case data describing possible faults, symptoms, verification procedures, and repairs. CaseBased reasoning (CBR) systems [15] typically store case histories in some form of data organisation, fronted by a reasoning system. New cases are investigated by presenting relevant case data to the system which then attempts to match this with recorded histories in order to identify similar cases which may provide relevant information.

\section{RELATED WORK}

Each of the information categories mentioned above has been widely used in systems for diagnosis and/or explanation generation in a variety of domains. A distinction can be made between systems that are essentially descriptive, i.e. producing documentation for the target domain, and those that provide a model of aspects of the domain that can be used for experiment and/or to reason about the domain. When dealing with complex structures, however, even systems which do not attempt to simulate behaviour will require to model the target domain in some detail.

A useful review of current work in model-based and qualitative reasoning is provided by Hunt, Lee and Price [9]. Different types of model include, in particular, structural models, which attempt to capture important physical or logical relationships of components of the target system, and functional models, which derive the operation of the system from information about the functional behaviour of its components. An example of an essentially structural model is that described by 
Xue, Yadav and Norrie [17] for use in building product design. This system also incorporates a knowledge-based system to generate building product descriptions automatically.

For our purposes, structural information is important: because the modelled product may be entirely new to the service agent, it will be essential to produce a model that he/she can readily relate to the real product, identifying components and their physical relationships. However, it will also be necessary, to describe operational procedures and for fault diagnosis, to build into the model representations of functional behaviour. Compositional modelling [7] derives a behavioural model from a knowledge base of the behaviour of components and their environment. Iwasaki et al [10] describe a web-based compositional modelling system, CDME, which formulates behaviour models of physical systems from domain knowledge. In CDME this knowledge is principally represented at two levels: an ontological level, which defines the vocabulary of the system, and a physical level, which describes aspects of behaviour in terms of this vocabulary.

The INT-OP project [1], concerned with the generation of operating procedures for chemical process plants, also incorporates elements of both descriptive and operational modelling. The system, CEP (Chemical Engineering Planner) produces plans which define sequences of actions to bring about specified changes in the plant. The basis of the system is a detailed model of the domain. Although the target domain of this project is more complex than the one we are considering, and the output more specialised, a number of elements of the work are relevant to our discussion. The plant model used defines a hierarchy of components and the connections between them, and a conclusion of the project was that the effort required to create this was substantial, and that tools to assist in domain development are vital. A further element of the model is a set of "pairs", defining associations between components. The significance of this in our context is that, for example, a fault identified in one component of the system may in fact be caused by a problem arising in a different component, with no close physical connection to the one under consideration.

A number of key issues emerge from the research described in the literature. The first is the need to integrate model-based aspects of the system with other elements. Hunt et al [9] observe that even in model-based diagnosis, much of the information that is necessary is not model-based, and includes data such as failure likelihoods for different components, and heuristics for fault localisation. Secondly, the need to derive information about a target product from data relating to its components calls for the use of intelligence in this integration. Finally, both these issues point to the central role carried by the organisational structure of data in the system. This needs to incorporate not only a detailed structural model of the target domain, but also behavioural information and representations of all the different kinds of information which may be called upon by the service agent. In the following section we outline the architecture of a system to meet these requirements. 


\section{SYSTEM ARCHITECTURE}

Figure 2 illustrates a top-level processing procedure of the proposed system.

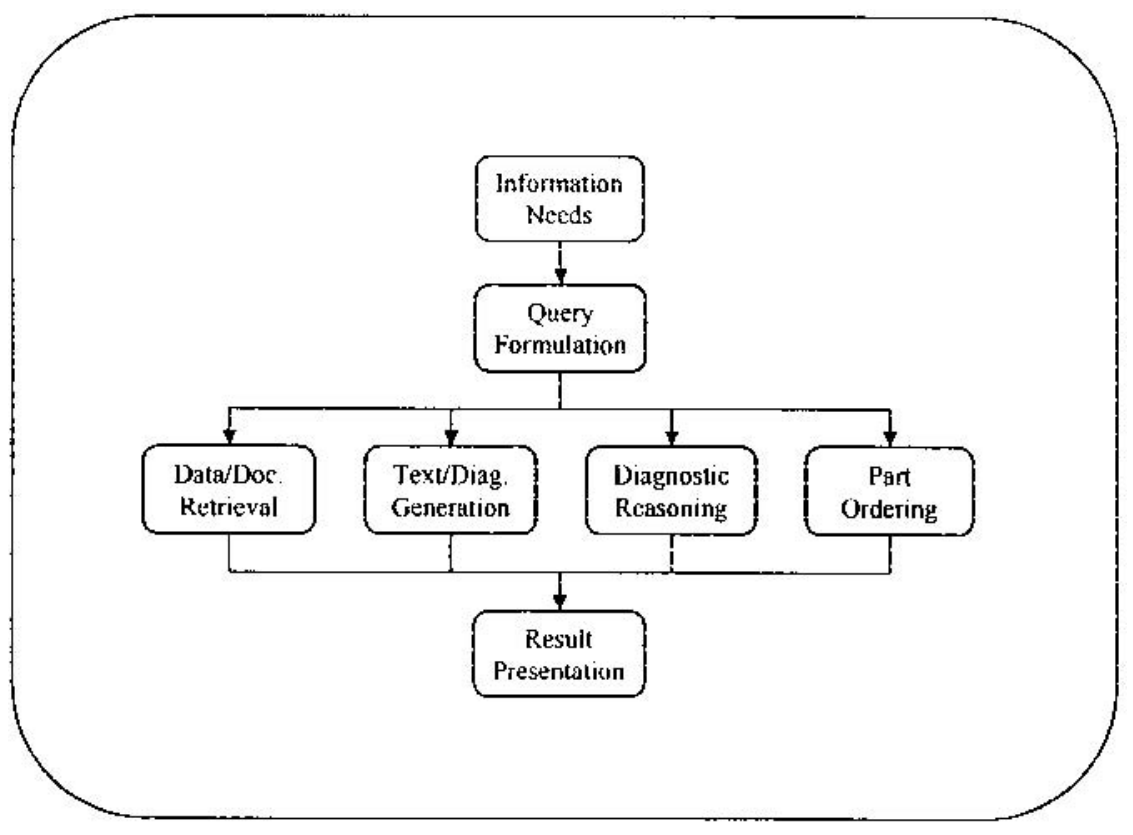

Figure 2. Processing procedure

Field engineers or other users raise Information Needs from their practical work. From these are formulated formal queries that the system can understand. According to the queries, one or more of the following could happen: Data/Document Retrieval, Text/Diagram Generation, Diagnostic Reasoning, or Part Ordering. Data/Document retrieval means finding some pre-stored data/documents which could satisfy the users needs; these could be of different media, such as text, image, audio, etc. Text/diagram generation uses text or diagrams for components of the product to create composite representations, for instance, 3D diagrams of products. Diagnostic reasoning addresses the other central aim of the system, to assist the engineer in fault diagnosis and repair. It may make use of techniques including casebased and rule-based systems to support this function. Finally, Part Ordering is provided to enable the engineer to obtain replacement parts when necessary. The result presentation takes the outcomes from the above processes for display to the user. Figure 3 shows a preliminary architecture for a system to support these processes. 


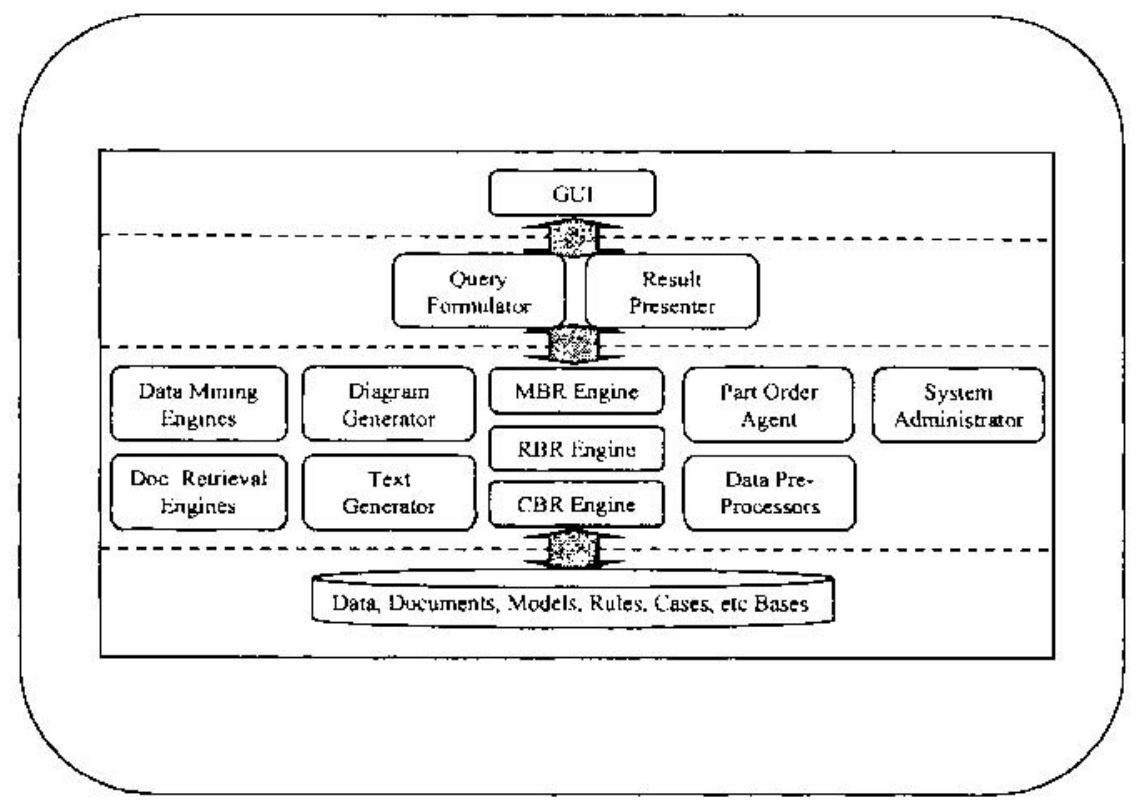

Figure 3. Integrated system framework

The system components included in this framework can be divided into four groups: User Interface (Graphic User Interface, User Requirement Formulator, Result Presenter); Processing Agents (Data/Document Retrieval Engines, Text/Diagram Generators, Diagnostic Reasoning Engines, Part Order Agent, etc); Administrative Agents (Data Pre-processors and System administrator); and Databases (data bases, document bases, model bases, rule bases, and case bases). Most of these components have a direct correspondence to the processes of Figure 3. The implementation envisaged represents system components in the form of servers or agents operating over the Internet; this could be refined with different kinds of architecture techniques, for example, Multi-Tier Client-Server and Multi-Agent Architectures. [6, 16].

\section{SYSTEM IMPLEMENTATION}

Figure 4 illustrates the architecture of the system implementation. This can be classified into four levels. From the left, the first is the client-side presentation, which includes pure HTML, Java Applets, and combinations of the two. This kind of presentation provides choices for graphical user interfaces across a company's Intranet or on the World Wide Web. Support for simple HTML means quicker 
prototypes, and support for a broader range of clients. Additionally, the Java plugin can be automatically downloaded to provide added applet support when necessary.

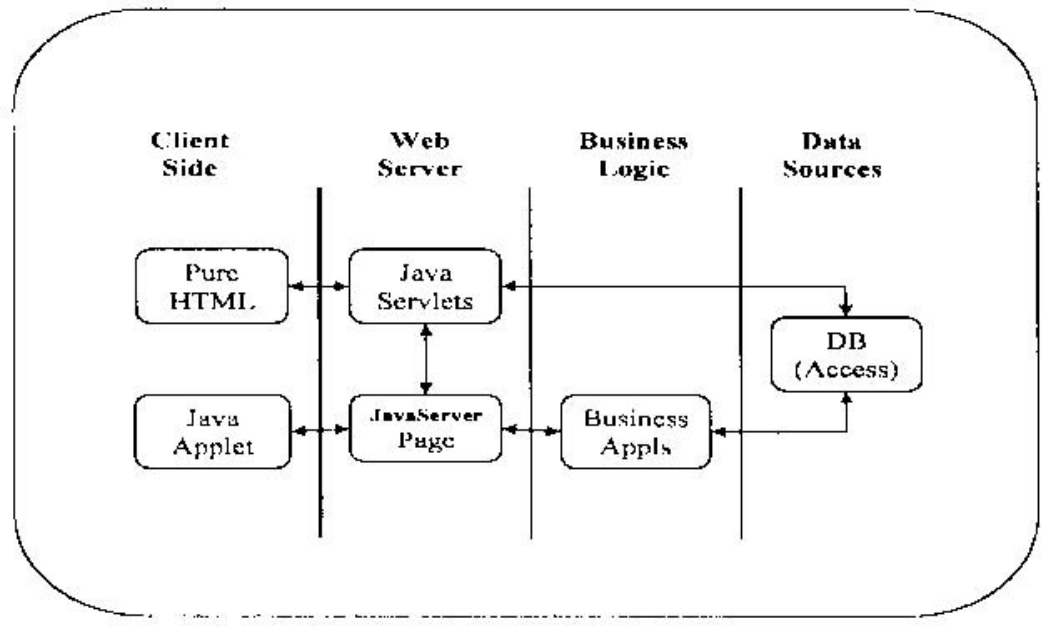

Figure 4. System Implementation architecture

At the second level is the Server-Side presentation, including Java Servlets and JavaServer Pages, and at the third level, Server-side business logic. The main technology here is JavaBeans. Enterprise JavaBeans (EJB) technology gives developers the ability to model objects by defining two distinct types of components: Session Beans and Entity Beans. Session Beans represent behaviours associated with client sessions - for example, a user purchase transaction - while Entity Beans represent collections of data, such as rows in a relational database, and encapsulate operations on the data they represent. Entity Beans are persistent, surviving as long as the data they are associated with remains viable. In this case, they provide a path to the final level, the database.

\section{DISCUSSION AND CONCLUSION}

The key issue identified in the architecture we have outlined is integration, which has two aspects: firstly, the integration of information relating to components of the product to create a composite picture, and secondly, the integration of the 
different forms of representation and modelling used. The first is the role of the diagram generator and text generator system components. The use of static "canned" information, however well-organised and presented, does not fully meet the demands of a flexible production process. The problem is, essentially, that the description of the product as a whole is more than a simple concatenation of the descriptions of its components. Dynamic explanation generation [13] can be used to reduce repetition of information and to produce a more coherent overall product description. This technique, however, applies only to text. A greater challenge is created by the need to pull together all the elements of the documentation of components, textual and other, into a single, integrated product description. This process may be seen as an aspect of product design, and appropriate design techniques, such as the use of CAD software, may have a role here.

The second major function of the system is to assist in the diagnosis of faults in service, and this is the principal purpose of the Model-Based, Rule-Based and CaseBased Reasoning components of the system. Operational simulation via a product model is particularly significant in this respect. Added intelligence may be provided by model-based reasoning [8], in which the model is manipulated by appropriate reasoning routines. A common approach is to use a "perfect" product model to generate a simulation of the correct behaviour of the system, and then to introduce faults to the model in an attempt to replicate the observed behaviour of the (faulty) real product. Model-based systems have in recent years found favour with industry, for example in the Tiger project [6]. Used in isolation, however, they suffer from the drawback that little or no explanation may be provided to supplement the observed model behaviour, so again, integration with the representational elements of the system is called for. Another goal is to integrate Knowledge-Based Systems with modelling techniques, including model-based reasoning and VR interfaces. Intelligent VR agents (IVRA) [2] adds intelligence to virtual reality to produce autonomous agents that model physical behaviour, using intelligence to guide the use of VR.

The Case-Based Reasoning element is also primarily to support fault diagnosis. CBR systems need not incorporate large databases; very often, a case base of some 100 previous histories will be sufficient to give useful pointers. In the present context, there are issues to be resolved concerning the matching of case data. Because the product under consideration may, in the extreme case, be unique, there may be no case histories relating to this particular model. Hence, it will be necessary for the CBR system to look for matches not just with the circumstances of the present case but also with the product model, in order to find, for example, cases in which a similar but not identical product has been involved. 


\section{REFERENCES}

1 Aylett, R., Petley, P., Chung, P.W.H., Soutter., J. and Rushton, A. Generating Operating Procedures for Chemical Process Plants. Integrated Manufacturing Systems: The International Journal of Manufacturing Technology Management. 1999; 6:328-42

2 Ballin, D. and Aylett, R. Intelligent Virtual Environment and Virtual Agents. Tutorial Material. $19^{\text {th }}$ SGES, 1999.

3 Buchanan, B.G. and Shortliffe, E.H. Rule Based Expert Systems - The MYCIN Experiments of the Stanford Heuristic Programming Project. Addison-Wesley, 1984.

4 Carey, R., Bell, G. and Marrin, C. The Virtual Reality Modeling Language (VRML97). ISO/IEC 14772-1. The VRML Consortium Inc, 1997.

5 Crowder, R.M., Wills, G., Heath I. and Hall, W. The Application of Hypermedia in the Factory Information Environment. Proc "FACTORY 2000" Conference; Cambridge: IEE Conference Publication 435, 1997.

6 Edwards, J. 3-Tier Client/Server at Work. Wiley, 1998.

7 Falkenhainer, B. and Forbus, K. Compositional Modeling: Finding the Right Model for the Job. Artificial Intelligence, 1991; 51: 1-3

8 Hunt, J.E, Lee, M.H. and Price, C.J. Progress in applying model based and qualitative reasoning to industrial applications. The MONET Newsletter, 1998; 1.

9 Hunt, J.E. Model Based and Qualitative Reasoning: Industrial Application. Proc 1st Int Workshop on Model-Based and Qualitative Reasoning - Perspectives for Industrial Applications; ECAI'96; Budapest; 1996.

10 Iwasaki, Y., Farquar, A., Fikes, R. and Rice, J. A Web-Based Compositional Modelling System for Sharing of Physical Knowledge. Proc 15th Int Joint Conf on AI; AAAI Press, 1997.

11 Milne, R., Nicol, C., Trave-Massuyes, L., Quevedo, J., Ghallab, M., Bousson, K., Dousson, C., Aguilar, J. and Guasch, A. TIGER: Real Time Situation Assessment of Dynamic Systems. Intelligent Systems Engineering Journal, 1994; 3.

12 Moulton, M. A Rule-Based Incident Tracking System. In Applications and Innovations in Expert Systems V, Macintosh, A. and Milne, R., eds. London: SGES Publications, 1997.

13 Rubinoff, R. Adapting Mumble: Experiences with Natural Language Generation. Proc AAAI-86; 1986.

14. Sowiztal, H., Rushworth, K. and Deening, M. The Java 3D API Specification. AddisonWesley, 1998.

15 Watson, I. Applying Case-Based Reasoning: Techniques for Enterprise Systems. London: Morgan Kaufman, 1997.

16 M. Wooldridge, N. R. Jennings, and D. Kinny. A Methodology for Agent-Oriented Analysis and Design. In Agents '99: Proceedings of the Third International Conference on Autonomous Agents, O. Etzioni, J. P. Muller, and J. Bradshaw, eds, Seattle, WA, May 1998.

17 Xue, D., Yadav, S. and Norrie, D.H. Development of an Intelligent System for Building Product Design and Manufacturing - Part1 : Product Modelling and Design. 1999 